Richtig kommunizieren, einfache Therapien

\title{
Das sind die Schlüssel für eine bessere Therapietreue bei COPD
}

— Um die Therapietreue von Patienten mit chronisch obstruktiver Lungenerkrankung (COPD) ist es nicht gut bestellt: $20 \%$ aller Rezepte werden nicht eingelöst und 30\% der verordneten Medikamente so falsch angewendet, dass daraus ernsthafte Gesundheitsschäden resultieren. Deshalb sind bei COPD Maßnahmen zur Verbesserung der Therapietreue wahrscheinlich wesentlich effektiver als jede Verbesserung der Medizin selbst, so Prof. Felix Herth, Thoraxklinik Heidelberg. Die Therapietreue lässt sich erhöhen durch kluge Medikamentenwahl: Einmalgabe statt Zweimalgabe täglich, einfache Anwendung, schneller Wirkeintritt, den der Patient sofort verspürt, sowie eine zuverlässige Wirkung über 24 Stunden.

Über diese Eigenschaften verfügt der neue Seebri ${ }^{\circledR}$ Breezhaler $^{\circledR}$ mit dem Wirkstoff Glykopyrronium, so Herth: Der lang wirksame Muskarin-Antagonist (LAMA) wirkt über 24 Stunden und muss nur einmal täglich inhaliert werden. Der Wirkeintritt erfolgt schnell: Bereits nach 5-15 Minuten bessert sich die FEV ${ }_{1}$ spürbar, so Herth. „Das hilft mir sofort", bemerken die Patienten.
Ein weiterer Vorteil des Breezhalers: Bereits ein geringer inspiratorischer Fluss genügt, um die Inhalation auszulösen. „Das ist ein Vorteil für Patienten mit deutlich eingeschränkter Lungenfunktion", sagte Herth.

In zwei Cross-Over-Studien gaben die Patienten an, den neuen Inhalator einfacher anwenden zu können. Klinisch ist Glykopyrronium $1 \mathrm{x} / \mathrm{d}$ dem Tiotropium 2 $\mathrm{x} / \mathrm{d}$ ebenbürtig bezüglich der Verbesserung der FEV 1 über 24 Stunden, der Verbesserung der inspiratorischen Kapazität (Reduktion der Überblähung) sowie der Verhinderung von Exazerbationen.

\section{Drei Säulen der Therapieadhärenz}

Noch wichtiger für die Therapietreue ist eine vertrauensvolle Arzt-Patienten-Kommunikation, die auch eine gute und wiederholte Schulung über die richtige Inhalation umfassen sollte. „Gute Kommunikation, angenehme Praxis-Atmosphäre, gutes Erinnerungs-Management - das sind die drei Säulen der Therapieadhärenz, erklärte Sascha Rombock, Geschäftsführer der Gesundheitsagentur keiro:denkt. „Gelungene

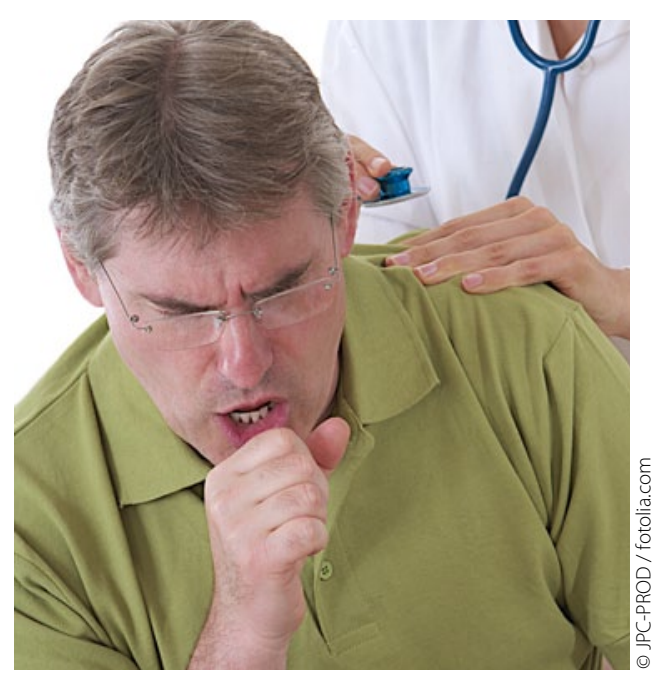

Husten, Auswurf, Atemnot - die belastende Trias bei COPD.

Kommunikation ist ein Hauptgrund der Patientenzufriedenheit", so Rombock: „Überzeugen Sie mit Inhalten, mit Sprache und mit Körpersprache, fragen Sie die Meinung des Patienten ab, sprechen Sie Gefühle an, erfragen Sie Gründe, loben und ermuntern Sie, dann erhöhen Sie die Wahrscheinlichkeit, dass der Patient ihre Therapie richtig anwendet."

- Dr. med. Dirk Einecke

Quelle: Presse-Vernissage „Therapietreue als Herausforderung bei COPD", Berlin, Februar 2013 (Veranstalter: Novartis)

\section{Nervosität, Abgeschlagenheit, Infektionsanfälligkeit Liegt ein Vitamin-B-Mangel vor?}

- B-Vitamine sind für eine gute Nervenfunktion wichtig. Während der Schwangerschaft und der Stillzeit, bei länger dauernden Stresssituationen, größeren sportlichen Leistungen oder in Wachstumsphasen besteht ein erhöhter Bedarf. Chronische Erkrankungen, die Einnahme bestimmter Medikamente oder Lebensgewohnheiten wie Rauchen oder der Konsum größerer Alkoholmengen erschweren eine ausreichende Versorgung an B-Vitaminen ebenso wie eine wenig ausgewogene Ernährungsweise - z. B. mit sehr proteinreicher Kost oder bei veganer Diät. Magen- oder Darmerkrankungen sind weitere Gründe, warum der Körper nicht genügend B-Vitamine aufnehmen oder metabolisieren kann.

Eine besondere Aufmerksamkeit bedarf die Vitamin-B-Versorgung bei Älteren: Hier kommt es durch Fehl- und Mangelernährung, durch Multimorbidität und damit verbundener Einnahme unterschiedlicher Medikamente sowie durch Malabsorption deutlich öfter zu Vitaminmangelerkrankungen.

Infolgedessen können schon in frühen Stadien unspezifische Symptome wie Leistungsabfall, Appetitlosigkeit, Nervosität auftreten. Bei schwerwiegenderen Defiziten kann es auch zu Polyneuropathien oder verringerten kognitiven Fähigkeiten kommen. Anämien, degenerative Demenz oder Depression können Folgen eines über einen längeren Zeitraum bestehenden Mangels sein. Bei sonst unauffälligen Befunden kann es ratsam sein, bereits bei körperlichen oder geistigen Leistungsdefiziten den Vitaminstatus des Patienten zu überprüfen und die entsprechenden Vitamine - beispielsweise mit Vitamin B-Komplex forte Hevert ${ }^{\oplus}$ - hoch dosiert zu substituieren.

- Red.

Quelle: nach Angaben des Unternehmens Hevert-Arzneimittel 